\title{
Application of Flipped Classroom Pedagogy to the Human Gross Anatomy Laboratory: Student Preferences and Learning Outcomes
}

\author{
Timothy R. Fleagle ${ }^{1}$, Nicholas C. Borcherding ${ }^{2}$, Jennie Harris ${ }^{3}$, and Darren S. Hoffmann ${ }^{1,}$ \\ ${ }^{1}$ Department of Anatomy and Cell Biology, Roy J. and Lucille A. Carver College of Medicine, \\ University of lowa, lowa City lowa \\ ${ }^{2}$ Medical Science Training Program, Roy J. and Lucille A. Carver College of Medicine, University \\ of lowa, lowa City, lowa \\ ${ }^{3}$ Department of Endodontics, University of lowa College of Dentistry, lowa City, lowa
}

\begin{abstract}
To improve student preparedness for anatomy laboratory dissection, the dental gross anatomy laboratory was transformed using flipped classroom pedagogy. Instead of spending class time explaining the procedures and anatomical structures for each laboratory, students were provided online materials to prepare for laboratory on their own. Eliminating in-class preparation provided the opportunity to end each period with integrative group activities that connected laboratory and lecture material and explored clinical correlations. Materials provided for prelaboratory preparation included: custom-made, three-dimensional (3D) anatomy videos, abbreviated dissection instructions, key atlas figures, and dissection videos. Data from three years of the course ( $n=241$ students) allowed for analysis of students' preferences for these materials and detailed tracking of usage of 3D anatomy videos. Students reported spending an average of 27:22 ( \pm 17:56) minutes preparing for laboratory, similar to the 30 minutes previously allocated for in-class dissection preparation. The 3D anatomy videos and key atlas figures were rated the most helpful resources. Scores on laboratory examinations were compared for the three years before the curriculum change $(2011-2013 ; n=242)$ and three years after $(2014-2016 ; n=241)$. There was no change in average grades on the first and second laboratory examinations. However, on the final semi-cumulative laboratory examination, scores were significantly higher in the post-flip classes $(P=0.04)$. These results demonstrate an effective model for applying flipped classroom
\end{abstract}

\footnotetext{
*Correspondence to: Dr. Darren Hoffmann, Department of Anatomy and Cell Biology, Carver College of Medicine, University of Iowa, 51 Newton Road, 1-402 BSB, Iowa City, IA 52242, USA. darren-hoffmann@uiowa.edu. NOTES ON CONTRIBUTORS

TIMOTHY R. FLEAGLE, B.A., is a first-year doctor of physical therapy graduate student and laboratory research assistant at The University of Iowa Roy J. and Lucille A. Carver College of Medicine, Iowa City, IA.

NICHOLAS C. BORCHERDING, M.S., is a fourth-year M.D./Ph.D. student at the Roy J. and Lucille A. Carver College of Medicine, Iowa City, Iowa. He is pursuing the Education Distinction Track as part of his medical doctorate and serves on the Medical Education Council and the Research Council for the University of Iowa.

JENNIE HARRIS, D.D.S., is a resident in the Department of Endodontics at the University of Iowa College of Dentistry, Iowa City, IA. She participated in survey data analysis for this project while she was a dental student.

DARREN S. HOFFMANN, Ph.D., is an assistant professor of anatomy and cell biology in the Department of Anatomy and Cell Biology at the University of Iowa Roy J. and Lucille A. Carver College of Medicine. Iowa City, Iowa. He is the course director for Dental Gross Anatomy and his research interests are in curriculum design, self-directed learning and integration of online resources into anatomy education.
} 
pedagogy to the gross anatomy laboratory and illustrate a meaningful role for 3D anatomy visualizations in a dissection-based course.

\section{Keywords}

gross anatomy education; medical education; flipped classroom; computer-aided instruction; cadaver dissection; gross anatomy laboratory; prelaboratory preparation; 3D anatomy; anatomy videos

\section{INTRODUCTION}

\section{Curriculum Context}

Health professional curricula are undergoing changes in response to transformations in the healthcare professional landscape and academic reports on medical education (Irby et al., 2010). In particular, integration of clinical and basic sciences is of critical importance, with curricula providing earlier clinical experiences and breaking down some of the disciplinary and clinical/basic science boundaries that were key features of the 1910 Flexner report (Flexner, 1910; Drake, 2014). This trend led to a shrinking of the typical two-year of the traditional basic science courses, including gross anatomy, and a fundamental reevaluation of the basic science competencies that should be achieved in the first and second years (Krane, 2016). This has impacted gross anatomy courses and cadaver dissection laboratories, as they are the largest blocks of curriculum time in the preclinical years (Drake et al., 2009). Consequently, gross anatomy course directors have reduced components of their courses (e.g., embryology) and developed innovative strategies to ensure that students can still experience dissection as a learning tool but with less time (e.g., rotating dissection) (McWhorter and Forester, 2004; Beale et al., 2013). These changes primarily focused on the lecture or laboratory dissection themselves, with comparatively less focus on how students prepare for laboratory dissection.

In order for learning to be effective in the gross anatomy laboratory, it is important that students are adequately prepared and knowledgeable in the dissection steps and anatomical structures that they will be uncovering in their work. Common assignments that may be given before the laboratory to help students prepare include readings from a dissection manual or key figures from an anatomy atlas. Reading assignments prior to class sessions have been frequently described in the literature as having low compliance rates (Burchfield and Sappington, 2000; Sappington et al., 2002; Clump et al., 2004). In anatomy laboratory, students who do not engage with the reading assignment or understand the readings/figures come to the laboratory with little knowledge of what to do first, or where their dissection is headed. When this occurs, dissection quality may be very poor, which has been correlated with decreased learning outcomes (Nwachukwu et al., 2015). To mitigate a preparation deficiency, faculty must either lead prelaboratory sessions to calibrate the whole group or provide dissection instructions throughout the period to unprepared students. 


\section{Flipped Classroom Pedagogy}

One pedagogical strategy that emphasizes efficiency and active learning is the flipped classroom approach. In this paradigm, students are assigned short videos and instructional materials to review prior to attending class (Yarbro et al., 2014; Abeysekera and Dawson, 2015). Multimedia formats are often emphasized because of increased completion of preclass assignments compared with reading assignments (Stelzer et al., 2009). To ensure compliance, a common feature of flipped pedagogy is a pre-session readiness assurance quiz (Nieder et al., 2005). The work done by students before class eliminates the need to address basic content in class and allows students to stay engaged during class time with activelearning activities (e.g., problem-solving, application, and group exercises) (Jensen et al., 2015). This presumably also saves students time later on in the learning cycle that they would have spent struggling on their own. Beyond impact on time and classroom structure, this pedagogical approach was proposed to reduce cognitive load by allowing students to better manage working memory during self-regulated study compared with traditional lectures (Abeysekera and Dawson, 2015). In their study, Abeysekera and Dawson (2015) proposed that flipped classroom pedagogy may increase students' competence, autonomy, and relatedness which improves intrinsic and extrinsic motivation. Flipped pedagogy was shown to be preferred by students over traditional methods and result in small but measureable increases in learning outcomes (Pierce and Fox, 2012; Gilboy et al., 2015). While the specific approach used in a course may vary depending on the context, flipped pedagogy requires providing quality materials before a session that allow students to achieve content attainment on their own. These materials can be curated or created by the instructor and are often in the form of videos that can be accessed via the internet. This method was recently applied to gross anatomy lecture classrooms (Lochner et al., 2016; Singh and Min, 2017).

The use of videos in higher education is prolific because they include the ability to self-pace, engage with content through prediction, manipulate models and select tasks, and reflect and respond to questions and prompts (Moreno and Mayer, 2007; Wouters et al., 2007). In practice, videos can have a powerful impact on orienting learners to a new subject (creating motivation to learn). Studies have illustrated that videos were perceived as "easier" media to learn from compared to primarily verbal content, lowering the barrier to entry on new topics (Marx and Frost, 1998), maximizing attention (Choi and Johnson, 2005), and improving learning outcomes and procedural skills (Holland et al., 2013; Mehrpour et al., 2013).

Videos of dissections have been implemented in gross anatomy courses in different ways with variable outcomes. Using dissection videos as a replacement for dissection, a study in a veterinary anatomy course demonstrated that dissection videos resulted in a negative impact on learning outcomes (Theoret et al., 2007). On the contrary, when used as an adjunct to lecture or laboratory instruction, student preferences and learning outcomes were more positive (Dilullo et al., 2006; Granger and Calleson, 2007; Al-Khalili and Coppoc, 2014; Choi-Lundberg et al., 2016). Taken together, these studies demonstrated the potential for dissection videos as resources for anatomy learning. However, these studies did not utilize video resources in a constructively-aligned flipped classroom structure. Further, the comparative value of other possible learning resources (e.g., atlas figures, written 
instruction, three-dimensional [3D] anatomy simulations) in a flipped classroom structure has not been evaluated.

The course described in this study was transformed using flipped pedagogy, such that students were provided with online prelaboratory materials (including dissection videos, key atlas figures, abbreviated written instructions and videos of 3D anatomy models), eliminating the need for in-class prelaboratory sessions. That time was instead used for advanced integrative group activities at the conclusion of the dissection period. The goal of this study was to evaluate the success of this approach on several dimensions. First, use of laboratory and prelaboratory time before and after the curriculum change was analyzed. Further, because videos of 3D anatomy models are a relatively new learning resource in anatomy, the usage of this resource was also tracked throughout the course. Second, it was hypothesized that students would prefer video-based resources for prelaboratory preparation. To evaluate this hypothesis, a mixed methods analysis was used to measure student opinions and preferences. Finally, it was also hypothesized that this approach would result in improved learning outcomes. To evaluate impact, a retrospective cohort study approach was utilized to evaluate outcomes on laboratory examinations in the three years before and three years after the curriculum change.

\section{MATERIALS AND METHODS}

\section{Course Context}

Course scope and assessment.-Human Gross Anatomy for Dental Students was a course for first year students in the second semester of the Doctor of Dental Surgery (DDS) program at the University of Iowa College of Dentistry. The course utilized lectures, interactive review sessions, and dissection of the human cadaver. Total contact hours for the course were 53 hours of classroom-based instruction and 72 hours of laboratory dissection. The content of the course included: gross anatomy of the trunk and upper limb, advanced gross anatomy of the head and neck, and basic neuroanatomy of the central nervous system. Regional dissections closely matched the lecture schedule and included the following regions: thorax, abdomen, back, upper limb, neck, and all head regions with additional dissections of the brain and spinal cord. The course was structured into five lecture units and three laboratory units that ran concurrently. Each lecture unit was assessed through a multiple-choice examination with items constructed in United States Medical Licensing Examination ${ }^{\circledR}\left(\mathrm{USMLE}^{\circledR}\right) / \mathrm{National}$ Board Dental Examination ${ }^{\circledR}\left(\mathrm{NBDE}^{\circledR}\right)$ style. Each laboratory unit was assessed through a steeplechase-style timed pin test wherein students were asked to identify 40 structures that were tagged on dissected cadaveric specimens. All laboratory examination questions were "what is the structure?" meaning that no functionbased questions were assessed in laboratory examinations. Over the six years of this study, the content and sequence of the course was unchanged. The instructional team was stable with the same course director throughout. While lecture examinations were virtually identical year-to-year, laboratory pin tests had to be constructed de novo each year using the dissected cadaveric specimens. Pin tests were constructed by the instructional team (three anatomy faculty) with a predetermined number of structures to be pinned per laboratory to ensure a similar distribution of content on each examination. All instructional team members 
completed each examination individually and group consensus was used to ensure consistent and appropriate difficulty of examination items. Laboratory practical items were scored as either right or wrong (no half-credit assigned) and spelling was graded leniently provided that the misspelled word could not be mistaken for another anatomical structure.

Dissection laboratories.-Dissection laboratories were identical throughout the study. Each week of the course had two cadaver dissections. Each dissection group of 6-7 students was divided into two teams, such that team A (3-4 students) dissected on Mondays and team B (3-4 students) dissected on Wednesdays. In each dissection period, the dissecting team completed the full dissection with faculty supervision and was responsible for finding a prescribed list of structures (laboratory objectives). The typical dissection period was 2.5 hours in length. At the conclusion of each dissection period, the non-dissecting team members joined the dissectors in the laboratory to go over what they found and reviewed the laboratory objectives. This peer teaching/learning period was 30 minutes in length. A similar rotating dissection method at the University of Iowa was described previously (Pizzimenti et al., 2016). Some advantages of this approach were to create free time for non-dissecting students and to reduce the number of students at each table during dissection without increasing the number of cadavers or faculty laboratory facilitators. The dissections used in this course were based on the dissections of the Grant's Dissector (Tank, 2012).

Prelaboratory preparation.-For the first three years of this study (2011-2013), students were assigned a set of pages from the dissection manual as at-home pre-reading. Compliance with this assignment was not assessed, and students were not asked to report how much time they spent on preclass reading. Because the instructional team found that student preparation before class was insufficient, each laboratory dissection began with a 30minute prelaboratory overview and orientation to the laboratory session given by the course director (author D.H.). This consisted of a short PowerPoint (Microsoft Corp, Redmond, WA) presentation of key atlas figures, a review of key dissection steps, and viewing of a dissection video. This activity was necessary to ensure that students were knowledgeable of the anatomy and dissection steps prior to starting the laboratory and could function autonomously during class. Once students arrived at the laboratory, they received hands-on support from two faculty facilitators (student-to-faculty ratio $=20: 1$ ).

An important first step in the course transformation process was to acknowledge that the reading assignments and live prelaboratory sessions were not working to prepare students. Evidence of this was found periodically in annual student evaluations of the course and in the authors' anecdotal observations of unprepared students making critical mistakes and requiring extensive direction. To more closely evaluate the adequacy of the new preparation method and assess the need for curriculum change, student opinions about prelaboratory preparation were gathered via a needs assessment survey that was administered anonymously to the 2013 class cohort. Briefly, this survey asked students if the classroombased prelaboratory sessions were sufficient to prepare them for dissection (yes/no), and what types of resources (anatomical knowledge and/or procedural knowledge) would be most beneficial. Of the 80 students in that class, 41 completed the survey (response rate of $51 \%)$. This survey instrument is provided in Supporting Information. 
The next step was to generate improved laboratory preparation materials. To that end, key images were curated from the anatomy atlases used in the course and a series of dissection videos produced in 2007 at the University of Iowa were utilized. The authors created a set of abbreviated dissection instructions and a series of narrated non-stereoscopic 3D anatomy videos. In addition, a series of integrative group activities for students to complete at the end of each laboratory were developed.

\section{Prelaboratory Materials in Flipped Design}

Traditional resources.-All of the resources provided in the in-class prelaboratories were provided for students to review online prior to their assigned dissections. First, the key atlas figures presented in the PowerPoint slides were provided to the students as a file to download. These were typically a set of 5-10 figures from the Grant's Atlas of Anatomy (Agur and Dalley, 2013) and Netter's Atlas of Anatomy (Netter, 2014) with only the key structures highlighted. The dissection steps that were explained in class were transformed into a series of approximately ten short sentences or bullet points that could be read and understood rapidly by all students (abbreviated instructions). Lastly, the dissection videos that were shown in class were posted online on the course website (Desire2Learn, Kitchener, Ontario, Canada). All resources were stored on the course website organized by each laboratory topic for easy access. Prelaboratory preparation resources were made available to the students one week prior to the scheduled laboratory. An example of the prelaboratory resources for one laboratory is provided in Supporting Information.

Three-dimensional anatomy prelaboratory video design.-The three-dimensional (3D) prelaboratory videos were designed using the presenter function in Cyber-Anatomy, version 3.4, a commercially-available product (Cyber-Science3D, Iowa City, IA). The videos were built by the course director to closely match the objectives of each laboratory. Each video was designed to be about 10 minutes in length, and the anatomy was presented in a way that mimicked the steps of the dissection process. At each key frame, labels were applied to the main structures that students were expected to identify throughout the dissection. The course director also recorded narration over the 3D animation to explain the relationships between the structures seen in the animation and minimize the amount of reading required for the students. Closed captions of the instructor narrations were added to the video files to allow for accessibility for hearing impaired students. The 3D anatomy laboratory videos were hosted on a Panopto Video Platform server (Panopto; Seattle, WA), which allowed for data collection on student use of this resource. Each access to a video was stored as a view, timestamped, and the duration of the view was recorded in minutes. In the Panopto server, students can view videos at faster or slower speeds, pause and skip within a video. View duration is reported according to the active view length normalized to $1 \mathrm{x}$ speed (e.g., watching a full 10-minute video at $2 \mathrm{x}$ speed is still recorded as a 10-minute view; watching two separate one-minute segments of a video in one viewing session would be reported as a two-minute view). To ensure that the analysis would not be skewed by short inconsequential views or repeated clicks, views of less than one minute in length were not included in the analysis. Multiple views within a 1-hour period were consolidated and considered to be one view. To compare viewership between videos without the confounding variable of video length, a secondary analysis was conducted in which video view durations 
for each video were normalized to the overall mean view duration of all videos (15:51 minutes). Other prelaboratory resources were also provided online (dissection video, abbreviated instructions, key figures PowerPoint slides), but these resources were not stored on a server that allowed for collection of data on student downloads or streaming views. All 20 of the 3D anatomy videos can be viewed online at the following public YouTube channel (YouTube, 2017).

Prelaboratory quiz.-In keeping with typical flipped classroom and team-based learning (TBL) pedagogies (Nieder et al., 2005), the course utilized "readiness assurance quizzes." All students who were dissecting were required to complete the associated prelaboratory quiz prior to entering the laboratory. These quiz scores were included in the overall course grade. Non-dissecting students were allowed to complete the prelaboratory quizzes if they chose to do so but their scores were not recorded. The questions on the prelaboratory quiz focused on the dissection steps and the anatomical structures and relationships to be discovered during the laboratory. The questions could be answered using any combination of the different resources provided (no single resource directly addressed all of the items on the quiz better than another resource). Questions were reviewed by course faculty to ensure that they were aligned with laboratory objectives. Students were permitted to submit multiple attempts on the quiz and only the highest attempt score was saved for the grade book. The average time that students spent on quizzes was collected from the learning management system. A sample of one of the prelaboratory quizzes is provided in Supporting Information.

\section{Group Activity Design}

Group activities were developed by the course director based on the unique goals of each laboratory. In some cases, the activities were designed to help students focus on a higherlevel concept that they frequently overlook (e.g., organizing their own bodies in the anatomical arrangement of the structures of the pharynx). In other cases, the activities were designed to help students integrate and translate the lecture material in their laboratory work (anatomical scavenger hunts based on functional clues). In still other cases, the activities were designed to help students prepare for examinations (mock pin tests) or apply their knowledge to clinical skills (practicing dental anesthesia). In all cases, the activities were designed to be fun and highly interactive, requiring involvement from all members of the team (both dissectors and non-dissectors). The tasks to be completed in the activities were often not difficult (should be able to be completed by the group in 15-20 minutes), but required simultaneous engagement with the dissection work and the underlying lecture and clinical concepts. A sample of one of the group activities is provided in Supporting Information.

\section{Course Data Collection}

Course data were collected from three years of the course prior to the implementation of the change in prelaboratory approach (2011-2013) and three years following implementation (2014-2016). Each class consisted of approximately 80 first year students, giving a combined $n=242$ for pre-intervention and $n=241$ for post-intervention. Class profiles across all six years of the study were similar. Demographic data were collected including age and gender, undergraduate academic achievement (cumulative grade point average and 
science grade point average), and the Academic Average (ACAD) and Perceptual Ability (PAT) subscores of the Dental Admission Test (DAT), a test required for admission to dental school in the United States and Canada (Table 1). Both ACAD and PAT subscores are reported on a scale of $1-30$. The national mean ACAD for admissions is 19 . The mean threshold for PAT varies between 16 and 18. Learning outcomes data was collected from the three laboratory examinations in the course. The first two examinations were typical unit examinations covering the following topics: Unit 1 - Trunk, Upper Limb and Neck; Unit 2 Head Basics and Neuroanatomy; and the third examination was a semi-cumulative examination that covered all material from the head and neck including deeper head and neck regions that were not in Unit 1 or 2 . The three years in each cohort were pooled and comparisons between the experimental conditions were analyzed using independent twotailed Student's $t$-test with significance set at $P<0.05$. In order to further assess the degree of variability in examination performance from year to year, mean and \pm SD for each year (sub-cohort) was calculated. Course performance data from three examinations from a separate cohort of 80 students was utilized to estimate the reliability of examination items using the laboratory examination construction method described above. Over the three examinations, the average Cronbach's $a$ was 0.701 with a range of $0.634-0.824$ using this laboratory examination setup approach. Because these examinations were constructed collaboratively by three experts, the examinations had high content validity. To further assess the criterion validity of the laboratory examinations, scores should be compared against a secondary assessment that measures a similar outcome. In this study, the closest available secondary assessment was performance on lecture examinations. The rank correlation between laboratory examinations and total lecture examination score for the course was calculated using Kendall's tau B. Tau was calculated and a was set to 0.05 for determining significance. Laboratory examination performance was significantly correlated with overall lecture examination performance $(P<0.05)$ for all of the laboratory examinations in the study. The average tau of all laboratory examinations for 2011-2013 (before prelaboratory change) was 0.402 (range: 0.320-0.545) and the average tau for 2014-2016 (after prelaboratory change) was 0.384 (range: $0.228-0.468$ ), indicating a moderate positive concordance between these assessments. Because the laboratory pin tests were created de novo each year on the dissected cadavers in the course, it was impossible to administer identical laboratory examinations from year to year. However, each year the examinations were constructed using the same method, and the instructional team attempted to keep the content distribution of the examinations as similar as possible. To compare the examination content distribution before and after the prelaboratory change, the number of pinned structures from each laboratory was determined for each examination. Mean numbers of pinned structures from each laboratory were calculated for the overall pre- and post-change cohorts. These were used to generate heatmaps to visualize similarity in content distribution. These heatmaps and examination keys for all six years of the study are provided in Supporting Information.

In order to assess student opinions on resource preferences and estimate time spent on prelaboratory work, a survey was conducted at the conclusion of the 2014 and 2015 courses. The survey was constructed by the authors, and reviewed by a small group of faculty and students to ensure clarity and discrimination between individuals with different opinions. 
The survey was administered anonymously online using the course management system (Desire2Learn) alongside the other course-end evaluations. The survey was completed by 133 of 161 students (response rate of $82.6 \%$ ). Reliability and validity analyses were conducted for items in the survey that addressed student opinions about importance of individual prelaboratory resources. Because there was no underlying assumption of similarity between student opinions regarding the different resources, the items related to the four resource types were each assessed separately. Cronbach's $a$ was used to estimate reliability. The average Cronbach's $a$ was 0.798 (range: 0.698-0.942). Because the survey utilized two items that addressed a similar construct (importance for preparation for laboratory work and importance for preparation for assessment), Kendall's tau B was used to assess the rank correlation between the two items for each resource. The average tau was 0.551 (range: $0.412-0.710$ ). Qualitative, free-response comments from the survey were subjected to analysis by two independent evaluators. All written comments were first analyzed using open coding and then placed into key concept groups. No theoretical framework was employed to filter the analysis. Each comment was tagged as related to the following topics: specific prelaboratory resources, the integrative group activities, or the laboratory/prelaboratory structure in general. Within each topic area, comments were sorted as positive, negative or suggestions for improvement. Major categories of comments were determined based on frequency. Concepts and categories were discussed by both reviewers and a subsequent round of axial coding was conducted to ensure that no important categories or concepts were missed. For the quantitative items in the survey, means and $\pm \mathrm{SD}$ were calculated and for items related to student preferences for prelaboratory resources, means were compared using one-way analysis of variance with the Benjamini, Krieger, and Yekutieli approach for multiple hypothesis correction $(q=0.05)$. The full survey is provided in Supporting Information.

Course data were collected by the course director, de-identified, and handled according to the protocols described in the University of Iowa approved IRB (\#201405827). All data analyses were conducted using GraphPad Prism, version 7 (GraphPad Software, Inc., LaJolla, CA).

\section{RESULTS}

\section{Use of Laboratory Time}

For the first three years of this study (pre-intervention), the first 30 minutes of each laboratory period was spent preparing students for the dissection portion of the laboratory. This does not include any self-directed study time that students spent reading the required dissection instructions. Although data on how much time spent preparing outside of class was not collected, it was expected that this would be highly variable. When asked if the classroom-based prelaboratory session was sufficient in the needs assessment survey, 26 of $41(63 \%)$ students said "No" indicating that better preparation was needed. When asked about the necessity of additional resources for anatomical and procedural knowledge, students indicated the importance of both knowledge domains with 36 of 40 (90\%) for anatomical knowledge and 31 of $38(82 \%)$ for procedural knowledge. These results 
prompted the curriculum change and supported the need to create and curate a variety of resources for prelaboratory preparation.

After implementing the change in curriculum, students in the 2014 and 2015 cohorts ( $n=$ 127) reported their average amount of time preparing for each laboratory. The mean of these self-reported averages was $27 \pm 18$ minutes dedicated to laboratory preparation (mean, \pm SD). Most of this time was spent watching the $3 \mathrm{D}$ anatomy videos and dissection videos per student comments. Because time spent on the online quizzes was captured by the learning management system, it was confirmed that the prelaboratory quiz was only a small fraction of students' time in prelaboratory preparation (average time per quiz attempt was 1 minute and 59 seconds). The quizzes were completed (achieved a score of 100\%) by most students in one or two attempts. All dissecting students completed their assigned prelaboratory quizzes prior to attending laboratory. The change from dissection preparation during the laboratory period to before the class period allowed students to choose their approach for laboratory preparation and enabled students to do more advanced integrative work during supervised class time (Fig. 1).

As the 3D anatomy videos were stored on an external server, precise usage data could be collected for this resource. The average number of views per student per video was 2.20 and the average video watch time per video was 15:51 minutes per student (Table 2). The videos that had more watch time than average were Pharynx and Disarticulation, Oral Cavity, Posterior Triangle of the Neck, and the Larynx. Most of the videos that were watched more than average were on dissections that were more difficult and had a strong regional connection to the practice of dentistry (Fig. 2, red bars). In this study, overall usage of the videos was in keeping with what was expected given the number of dissecting students in each laboratory. There were approximately 40 dissecting students in each laboratory session. If every dissecting student watched a video for a given laboratory for all three years in the study, there would be a total of 120 unique users for that video. Most videos had between 110 and 140 unique users. Those videos with more than 120 unique users must have also been viewed by the non-dissecting students, either in preparation for peer teaching or preparation for later assessment in the course. Those with fewer than 120 unique users were not used by all of the dissecting students in the study. Most videos were near or above 120 unique users, and average views and minutes exceeded the length of the videos. This was an indication that the resource was effective for most students and most laboratories.

\section{Student Opinions and Resource Preferences}

Students were polled on their opinions about the in-class group activities in order to determine if students perceived them to be relevant to key laboratory objectives and achievable in the allotted time. Of respondents, $88.63 \%$ reported that they agreed or strongly agreed with the statement that the group laboratory activities helped emphasize important concepts from the laboratory ( $4.21 \pm 0.769$, mean $\pm \mathrm{SD}, n=132)$ on a five- point Likert agreement scale $(1=$ Strongly Disagree, $5=$ Strongly Agree $)$. Of respondents, $80.3 \%$ agreed or strongly agreed with the statement that the group laboratory activities were achievable by all members of the team during the peer teaching/learning session $(4.06 \pm 0.923, n=132)$. 
Students were asked to evaluate all four of the provided prelaboratory resources in terms of their importance for preparing for laboratory work. Two of the resources were particularly highly rated as useful for the work: key atlas figures and 3D anatomy videos. For these two resources, approximately $90 \%$ of students indicated that these resources were important or very important, with very similar average scores. Student ratings for these two resources were significantly elevated compared to both the dissection videos and abbreviated instructions. The abbreviated instructions were also rated significantly higher than the dissection videos (Fig. 3).

These quantitative results were supported by the free-response commentary provided by students in the opinion survey (themes summarized in Table 3). Qualitative analysis revealed that student opinions about 3D anatomy videos were positive, indicating that the resource provided good preparation for both dissection procedure and anatomical relationships. Dissection videos were also cited as being useful for understanding dissection procedure, but not for anatomical relationships. Both 3D anatomy videos and key figures were frequently cited as being useful for preparation for later assessments in the course. Dissection videos were indicated as having problems with visual clarity and variability in technique compared to written instructions. Group activities were cited as helpful for emphasizing important anatomical concepts, and holding students to the task of working through difficult material. However, students indicated that the difficulty level may have been too high for newly learned content and there was frequently an uneven level of participation within a group. Students recommended that non-dissecting team members should be more prepared or their participation should be enforced in group activities. Overall, students indicated that the online prelaboratory structure provided needed flexibility in terms of time and location as well as diversity of available resources. Finally, prelaboratory resources were indicated to be of the best quality when they reinforced anatomical concepts and were linked to the dissection procedure.

\section{Class Performance}

To determine if the implementation of a flipped prelaboratory would have any impact on student achievement in the class, academic achievement on laboratory examinations was assessed (the most closely related outcome to the laboratory preparation process). Examination performance on the first and second examinations was indistinguishable between the three years before the curriculum change and the three years after the change (examination 1 Cohen's $d=0.044$; examination 2 Cohen's $d=0.102$ ). However, scores for the third laboratory examination were significantly higher in the post-change group although the effect size was small (Cohen's $d=0.184$ ) (Table 4). In order to investigate the degree of variation from class to class, the means and standard deviations of each sub-cohort (year) were analyzed. The results showed that examination performance within the pre- and postintervention cohorts had variation from year to year. These data strongly supported pooling of multiple years of data in order to assess impact on learning outcomes and applying a conservative interpretation to the data. 


\section{DISCUSSION}

The results of this study illustrated that flipping the anatomy laboratory created time for integrative group activities in class. It resulted in laboratory work and academic performance outcomes that were at a similar or higher level as compared to preparation using a traditional classroom-based prelaboratory session. Data was not collected to assess quality of laboratory work after the change in prelaboratory structure; however, the instructional team did observe anecdotal positive changes in the preparedness of dissecting students. This led to a less directive and more collaborative relationship between the dissecting students and faculty. Of the different prelaboratory materials that were provided, the 3D anatomy videos and key atlas images were evaluated as most helpful.

This study extended the findings of Findlater and colleagues who described using recorded prepractical lectures as prelaboratory preparation leading to positive learning outcomes (Findlater et al., 2012). These findings also added to the growing body of research on use of dissection videos as an outside-of-class learning resource (Dilullo et al., 2006; Granger and Calleson, 2007; Al-Khalili and Coppoc, 2014; Choi-Lundberg et al., 2016). A prior study of dissection videos by Mahmud et al., however, demonstrated a non-significant effect on learning outcomes (Mahmud et al., 2011). Differences in context, including accessibility to resources, may have contributed to the discrepancy between this previous study and the current one. Streaming dissection videos were used by DiLullo et al., and data from that study demonstrated strong support from students and perceived benefits for learning (DiLullo et al., 2006).

The current study had only a modest impact on learning outcomes (2\%, which corresponds to 1 additional question on the examination), and the change was only significant in the last examination of the course. A similar result was reported in a study of dissection videos in a condensed curriculum (Topping, 2014). The last examination in this course was comprehensive of head and neck anatomy, and included more content than either of the two previous examinations. This may have created the conditions necessary to detect an impact of the curriculum enhancement. This examination also included regions of the body that had been dissected long before the final examination (i.e., neck regions). These regions were often contextually different after completing other dissections later in the course, such as disarticulation of the craniovertebral joints. In addition, this examination addressed on regions that were difficult to dissect and understand spatially (e.g., nasal cavity, sublingual region, etc.). It is possible that when the students came back to study the neck in preparation for the final examination, the availability of additional laboratory resources allowed them to make better sense of these most unfamiliar structures. It is important to acknowledge that it is challenging to determine the source of this modest change in learning outcomes. While the student population and laboratory examinations were very similar year-to-year, the curriculum change had several dimensions that may have impacted learning outcome: the availability of increased/better prelaboratory resources, possibly improved laboratory dissections, or improved learning due to the addition of integrative group activities at the end of each laboratory. 
The videos on the neck regions had higher numbers of views and view duration compared to overall averages across all of the videos. Further, the laboratories on pharynx, nasal cavity, oral cavity, and larynx had the highest numbers of unique users and average minutes/user. These videos may be more heavily watched because of (a) the difficulty of the material; (b) greater interest to this particular student population because of their dental relevance; and (c) pressure to perform better on the last examination for final course grades. Nonetheless, the examination that demonstrated a positive change in learning outcomes is also the examination that covered topics that had the highest usage of prelaboratory learning materials, suggesting a link between usage and learning outcome. This suggestion is supported by Saxena et al., wherein the authors reported that students who used the videos more demonstrated a small but measureable increase in course performance (Saxena et al., 2008).

The 3D anatomy videos were the only fundamentally new resource that had not been previously used in the face-to-face prelaboratories. For this reason, and because of the work required to create these $3 \mathrm{D}$ animations, the authors were especially interested in understanding how students used this resource. It has been previously demonstrated that $3 \mathrm{D}$ anatomy visualizations have a significant positive effect on spatial knowledge acquisition and user satisfaction when compared with 2D images (Yammine and Violato, 2015). Further, 3D anatomy software is a popular and accessible resource for anatomy students at all levels (i.e., Visible Body, Netter Interactive, etc.). However, the research on pedagogical strategies for using them in health professions curricula is limited (Hoyek et al., 2014; Attardi and Rogers, 2015). The results of the current study demonstrate a novel pedagogical application of 3D anatomy videos into a dissection-based anatomy course.

Viewership of the 3D anatomy videos was high and consistent throughout all years of the study. This observation is consistent with two previous studies on student usage of anatomy dissection videos (Saxena et al., 2008; Choi-Lundberg et al., 2016). However, the degree of usage reported in these studies was at a lower level than what was seen here. This may have been due to the deliberate integration of the prelaboratory materials toward a required prelaboratory quiz in the current study. Further research will be necessary to evaluate how viewing of this resource corresponds to preparation for laboratory dissection vs. preparation for assessment. Students preferred 3D anatomy videos and key atlas figures, which was a similar result as described by Choi-Lundberg et al., however in that study comparisons were only made against other class components, not other laboratory preparation resources (ChoiLundberg et al., 2016). Most research on prelaboratory resources to-date has focused on use of dissection videos or photographs for prelaboratory preparation (Al-Khalili and Coppoc, 2014; Choi-Lundberg et al., 2016). Yet in this study, students clearly demonstrated a preference for 3D animated videos as opposed to dissection videos. Another interesting finding was that the 3D anatomy videos and the key atlas figures were both rated at similar high levels. While there was no data to support a reason for the result, these two resources had a common visual presentation. Further research will be necessary to fully describe the qualities that make a prelaboratory resource effective and differentiate it from the resources used in this study. 
Students expressed a high degree of satisfaction with this transformation of the course, in spite of the fact that it created more structured "homework" for them prior to laboratories. This could be due to several factors. Anatomy dissection is often seen as a positive part of the course and taking time away from the laboratory with dry, in-class prelaboratory sessions could be demotivating for students. Further, in-class prelaboratories do nothing to encourage students to take responsibility for their own learning, one of the important tenets of adult learning theory (Knowles et al., 2005). In this flipped model, students had more responsibility for each laboratory experience, which may have led to increased effort, satisfaction and confidence. It is important to consider whether the curriculum approach used in this study did, in fact, fit a strict pedagogical definition of the flipped classroom, given that the flipped classroom is more commonly applied to lecture-based courses, and not necessarily dissection-based laboratories. The intervention described in the current study resulted in moving lecture-based prelaboratory instruction into the online space for students to complete prior to coming to class. This time was then converted into active learning activities that were completed through group discussion. In this sense, the intervention was very consistent with flipped pedagogy. However, in this study, pre-course work and active learning were separated by a dissection period that was largely unchanged in the preintervention and post-intervention cohorts. It was not clear whether students in the postintervention cohort made meaningful connections between the prelaboratory preparation work and the engaged group learning activities. Furthermore, it was unknown whether this transformation resulted in decreased study time after the laboratory sessions, which is a commonly held, but not commonly measured assumption about flipped classroom design. Nonetheless, this curriculum transformation was inspired by the tenets of flipped classroom pedagogy, and the result of the change was increased opportunities for active learning.

\section{Limitations of This Study}

One major limitation of this study was that detailed usage data was only available for the 3D anatomy videos. Another limitation was the need to create resources tailored to the dissection approach. Many anatomy instructors create their own custom dissection approaches to suit their students and program needs. Thus, the resources that were created for this course may not be well-suited for other institutions. This was emphasized by comments from students indicating that an important quality of the videos was that they emphasized critical dissection steps. Creating custom video resources may not be an achievable curriculum change goal for all anatomy faculty. However, the work in this study certainly demonstrated that the effort required to take on this transformation did result in student satisfaction, more advanced work in the anatomy laboratory, and, in the case of the comprehensive final examination, modest improvement in learning outcomes.

A final limitation of this study was that time on task and cognitive load were not precisely measured before or after the curriculum change. Because it was unknown how much time the pre-flip cohort students spent completing prelaboratory reading assignments as well as compliance rates for pre-laboratory reading, it was unknown whether the impact of this transformation was due to improved quality of work, increased time on task or both. The impact on time on task was likely variable depending on the student, based on prior research indicating that compliance for prelaboratory reading assignments was mixed within classes 
(Burchfield and Sappington, 2000; Sappington et al., 2002; Clump et al, 2004). The prelaboratory preparation involved similar knowledge expectations before and after the change in prelaboratory approach. However, the main difference was that students in the post-change cohort conducted all of their preparation in a self-regulated manner. This typically reduces cognitive load because students can control pace and select resources appropriate to their learning needs (Clark et al., 2005). On the other hand, students who did self-regulated laboratory preparation also had an additional resource to select from (3D anatomy videos) and completed a short quiz before each laboratory. These factors may have increased cognitive load, in spite of their constructive alignment. Further study in a more controlled experimental context will be necessary to examine the impact of self-regulated prelaboratory preparation on learning dynamics and the relationship of cognitive load to learning outcomes.

\title{
CONCLUSIONS
}

This study illustrated a method for instructor-supported, self-regulated prelaboratory preparation that resulted in learning outcomes similar to a classroom-based approach. This self-regulated approach was supported by feedback of students who had clear preferences for the types of resources they chose to utilize for this activity. Finally, this study demonstrated a meaningful and specific role for 3D anatomy visualizations in the modern anatomy curriculum.

\section{Supplementary Material}

Refer to Web version on PubMed Central for supplementary material.

\section{ACKNOWLEDGMENTS}

\begin{abstract}
The authors declare that there are no conflicts of interests regarding the publication of this paper. Dr. Darren Hoffmann was a paid consultant for Cyber-Anatomy, Inc. (now VIVED), assisting in the development of this software. However, he received no compensation for sales of this product. Cyber-Anatomy software was used independently by the author to develop the 3D Anatomy videos in this study, and research on these videos and the curriculum change was conducted without the involvement of Cyber-Anatomy, Inc. The authors wish to acknowledge the generous assistance of Dr. Martine Dunnwald in the editing of this manuscript. The authors also acknowledge The University of Iowa College of Dentistry and Office of Teaching and Learning and Technology for their advice and consultation throughout this project. This work was supported by an internal grant from the University of Iowa Academic Technology Advisory Council and by NIH F30 CA206255 (NB).
\end{abstract}

Grant sponsor: Ruth L. Kirschstein Individual Predoctoral National Research Service Award (NRSA) for MD/PhD (NIH F30) (awarded for N.B.); contract grant number: CA206255

Grant sponsor: University of Iowa Academic Technology Advisory Council Innovations in Teaching with Technology Award (awarded to D.H.)

\section{LITERATURE CITED}

Abeysekera L, Dawson P. 2015 Motivation and cognitive load in the flipped classroom: Definition, rationale and a call for research. High Educ Res Dev 34:1-4.

Agur AM, Dalley AF. 2013 Grant's Atlas of Anatomy. 13th Ed. Philadelphia, PA: Lippincott Williams \& Wilkins $871 \mathrm{p}$. 
Al-Khalili SM, Coppoc GL. 2014 2D and 3D stereoscopic videos used as pre-anatomy lab tools improve students' examination performance in a veterinary gross anatomy course. J Vet Med Educ 41:68-76. [PubMed: 24418924]

Attardi SM, Rogers KA. 2015 Design and implementation of an online systemic human anatomy course with laboratory. Anat Sci Educ 8:53-62. [PubMed: 24920278]

Beale EG, Tarwater PM, Lee VH. 2013 A retrospective look at replacing face-to-face embryology instruction with online lectures in a human anatomy course. Anat Sci Educ 7:234-241. [PubMed: 23959807]

Burchfield CM, Sappington J. 2000 Compliance with required reading assignments. Teach Psychol 27:58-60.

Choi HJ, Johnson SD. 2005 The effect of context-based video instruction on learning and motivation in online courses. Am J Dist Educ 19:215-227.

Choi-Lundberg DL, Cuellar WA, Williams AM. 2016 Online dissection audiovisual resources for human anatomy: Undergraduate medical students' usage and learning outcomes. Anat Sci Educ 9:545-554. [PubMed: 27802370]

Clark RC, Nguyen F, Sweller J. 2005 Efficiency in Learning: Evidence-Based Guidelines to Manage Cognitive Load. 1st Ed. San Francisco, CA: Pfeiffer 389 p.

Clump MA, Bauer H, Bradley C. 2004 The extent to which psychology students read textbooks: A multiple class analysis of reading across the psychology curriculum. J Instr Psychol 31:227-232.

DiLullo C, Coughlin P, D’ Angelo M, McGuinness M, Bandle J, Slotkin EM, Shainker SA, Wenger C, Berray SJ. 2006 Anatomy in a new curriculum: Facilitating the learning of gross anatomy using web access streaming dissection videos. J Vis Commun Med 29:99-108. [PubMed: 17162338]

Drake RL, McBride JM, Lachman N, Pawlina W. 2009 Medical education in the anatomical sciences: The winds of change continue to blow. Anat Sci Educ 2:253-259. [PubMed: 19890982]

Drake RL. 2014 A retrospective and prospective look at medical education in the United States: Trends shaping anatomical sciences education. J Anat 224: 256-260. [PubMed: 23600681]

Findlater GS, Kristmundsdottir F, Parson SH, Gillingwater TH. 2012 Development of a supported selfdirected learning approach for anatomy education. Anat Sci Educ 5:114-121. [PubMed: 22223487]

Flexner A 1910 Medical Education in the United States and Canada A Report to the Carnegie Foundation for the Advancement of Teaching. Carnegie Bulletin 4. 1st Ed.Boston, MA: D.B. Updike, The Merrymount Press 336 p.

Gilboy MB, Heinerichs S, Pazzaglia G. 2015 Enhancing student engagement using the flipped classroom. J Nutr Educ Behav 47:109-114. [PubMed: 25262529]

Granger NA, Calleson D. 2007 The impact of alternating dissection on student performance in a medical anatomy course: Are dissection videos an effective substitute for actual dissection? Clin Anat 20:315-321. [PubMed: 16795027]

Holland A, Smith F, McCrossan G, Adamson E, Watt S, Penny K. 2013 Online video in clinical skills education of oral medication administration for undergraduate student nurses: A mixed methods, prospective cohort study. Nurse Educ Today 33:663-670. [PubMed: 22341996]

Hoyek N, Collet C, Di Rienzo F, De Almeida M, Guillot A. 2014 Effectiveness of three-dimensional digital animation in teaching human anatomy in an authentic classroom context. Anat Sci Educ 7:430-437. [PubMed: 24678034]

Irby DM, Cooke M, O'Brien BC. 2010 Calls for the reform of medical education by the Carnegie Foundation for the advancement of teaching: 1910 and 2010. Acad Med 85:220-227. [PubMed: 20107346]

Jensen JL, Kummer TA, d M Godoy PD. 2015 Improvements from a flipped classroom may simply be the fruits of active learning. CBE Life Sci Educ 14:ar5. [PubMed: 25699543]

Knowles MS, Holton EF III, Swanson RA. 2005 The Adult Learner: The Definitive Classic in Adult Education and Human Resource Development. 6th Ed. Burlington, MA: Elsevier 378 p.

Krane NK. 2016 Curriculum Inventory in Context Length of Academic Levels Distributions in US and Canadian Medical Schools: 2015-2016. 1st Ed. Washington, DC: American Association of Medical Colleges 3 p. URL: https://www.aamc.org/download/464758/data/ciic03-1jan2016.pdf [accessed 12 April 2017]. 
Lochner L, Wieser H, Waldboth S, Mischo-Kelling M. 2016 Combining traditional anatomy lectures with e-learning activities: How do students perceive their learning experience? Int J Med Educ 7:69-74. [PubMed: 26897012]

Mahmud W, Hyder O, Butt J, Aftab A. 2011 Dissection videos do not improve anatomy examination scores. Anat Sci Educ 4:16-21. [PubMed: 21265032]

Marx RD, Frost PJ. 1998 Toward optimal use of video in management education: Examining the evidence. J Manag Dev 17:243-250.

McWhorter DL, Forester JP. 2004 Effects of an alternate dissection schedule on gross anatomy laboratory practical performance. Clin Anat 17:144-148. [PubMed: 14974103]

Mehrpour SR, Aghamirsalim M, Motamedi SM, Ardeshir Larijani F, Sorbi R. 2013 A supplemental video teaching tool enhances splinting skills. Clin Orthop Relat Res 471:649-654. [PubMed: 23054528]

Moreno R, Mayer R. 2007 Interactive multimodal learning environments. Educ Psychol Rev 19:309_ 326.

Netter FH. 2014 Atlas of Human Anatomy. 6th Ed. Philadelphia, PA: Saunders/Elsevier 640 p.

Nieder GL, Parmelee DX, Stolfi A, Hudes PD. 2005 Team-based learning in a medical gross anatomy and embryology course. Clin Anat 18:56-63. [PubMed: 15597377]

Nwachukwu C, Lachman N, Pawlina W. 2015 Evaluating dissection in the gross anatomy course: Correlation between quality of laboratory dissection and students outcomes. Anat Sci Educ 8:4552. [PubMed: 24799441]

Pierce R, Fox J. 2012 Vodcasts and active-learning exercises in a "flipped classroom" model of a renal pharmacotherapy module. Am J Pharm Educ 76:196. [PubMed: 23275661]

Pizzimenti MA, Pantazis N, Sandra A, Hoffmann DS, Lenoch S, Ferguson KJ. 2016 Dissection and dissection-associated required experiences improve student performance in gross anatomy: Differences among quartiles. Anat Sci Educ 9:238-246. [PubMed: 26536279]

Sappington J, Kinsey K, Munsayac K. 2002 Two studies of reading compliance among college students. Teach Psychol 29:272-274.

Saxena V, Natarajan P, O'Sullivan PS, Jain S. 2008 Effect of the use of instructional anatomy videos on student performance. Anat Sci Educ 1:159-165. [PubMed: 19177403]

Singh A, Min AK. 2017 Digital lectures for learning gross anatomy: A study of their efficacy. Korean J Med Educ 29:27-32. [PubMed: 28264551]

Stelzer T, Gladding G, Mestre JP, Brookes DT. 2009 Comparing the efficacy of multimedia modules with traditional textbooks for learning introductory physics content. Am J Phys 77:184-190.

Tank PW. 2012 Grant's Dissector. 15th Ed. Philadelphia, PA: Lippincott, Williams \& Wilkins 288 p.

Theoret CL, Carmel EN, Bernier S. 2007 Why dissection videos should not replace cadaver prosections in the gross veterinary anatomy curriculum: Results from a comparative study. J Vet Med Educ 34:151-156. [PubMed: 17446641]

Topping DB. 2014 Gross anatomy videos: Student satisfaction, usage and effect on student performance in a condensed curriculum. Anat Sci Educ 7:273-279. [PubMed: 24106107]

Wouters P, Tabbers HK, Paas F. 2007 Interactivity in video-based models. Educ Psychol Rev 19:327342.

Yammine K, Violato C. 2015 A meta-analysis of the educational effectiveness of three-dimensional visualization technologies in teaching anatomy. Anat Sci Educ 8:525-538. [PubMed: 25557582]

Yarbro J, Arfstrom KM, McKnight K, McKnight P. 2014 Extension of a Review of Flipped Learning. 1st Ed. Fairfax, VA: Flipped Learning Network, Pearson, George Mason University 20 p. URL: http://flippedlearning.org/wp-content/uploads/2016/07/Extension-of-FLipped-Learning-LItReview-June-2014.pdf [accessed 12 April 2017].

YouTube. 2017 Dental Gross Anatomy Laboratory Videos. University of Iowa, Iowa City, Iowa URL: http://yt.vu/+uiowagristo [accessed 13 June 2017]. 
Preparation: Reading Assignments

2011-2013

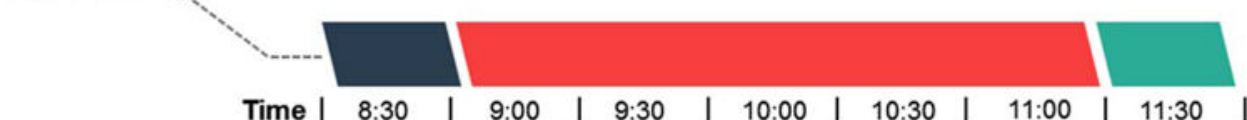

2014-2016

Preparation: Video Assignment and Pre-quiz

\begin{tabular}{l} 
Prelab Dissection $\quad$ Peer Teaching Integration Activity \\
\hline
\end{tabular}

Figure 1.

Graphical representation of course changes before and after change in prelaboratory preparation. Laboratory session start and end times were consistent (8:30-12:00). 


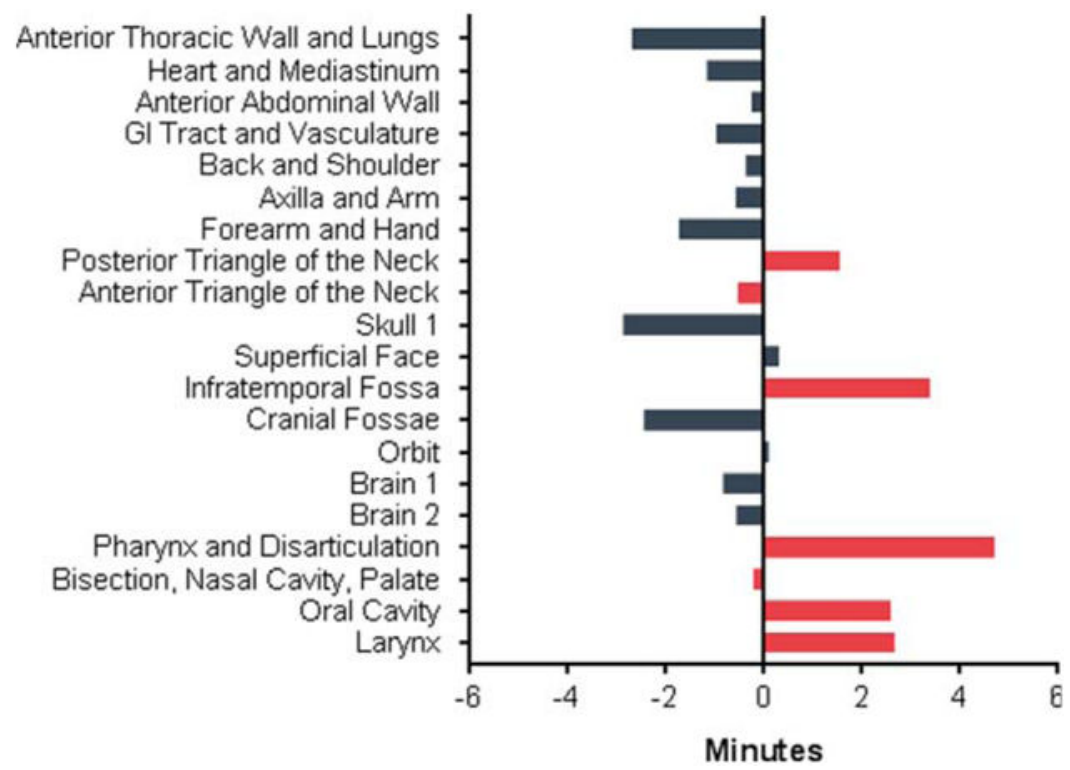

Figure 2.

Mean-centralized video duration averages for individual videos. Each video duration was normalized to the mean view duration of all videos (15:51, vertical line at 0.0). 


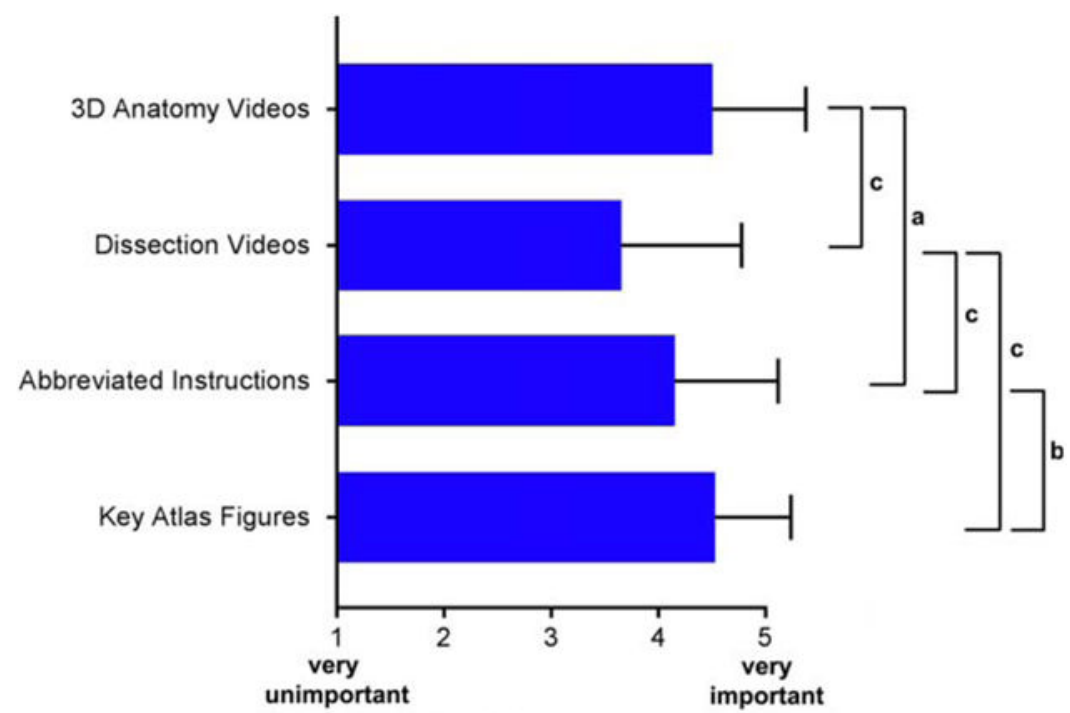

Likert Scale Response

Figure 3.

Mean responses on a Likert (1-5) scale when students were asked how important prelaboratory resources were to prepare for laboratory $(1=$ very unimportant, $3=$ neutral, 5 $=$ very important $) ;{ }^{\mathrm{a}} P=0.002 ;{ }^{\mathrm{b}} P=0.001 ;{ }^{\mathrm{c}} P<0.001$. 


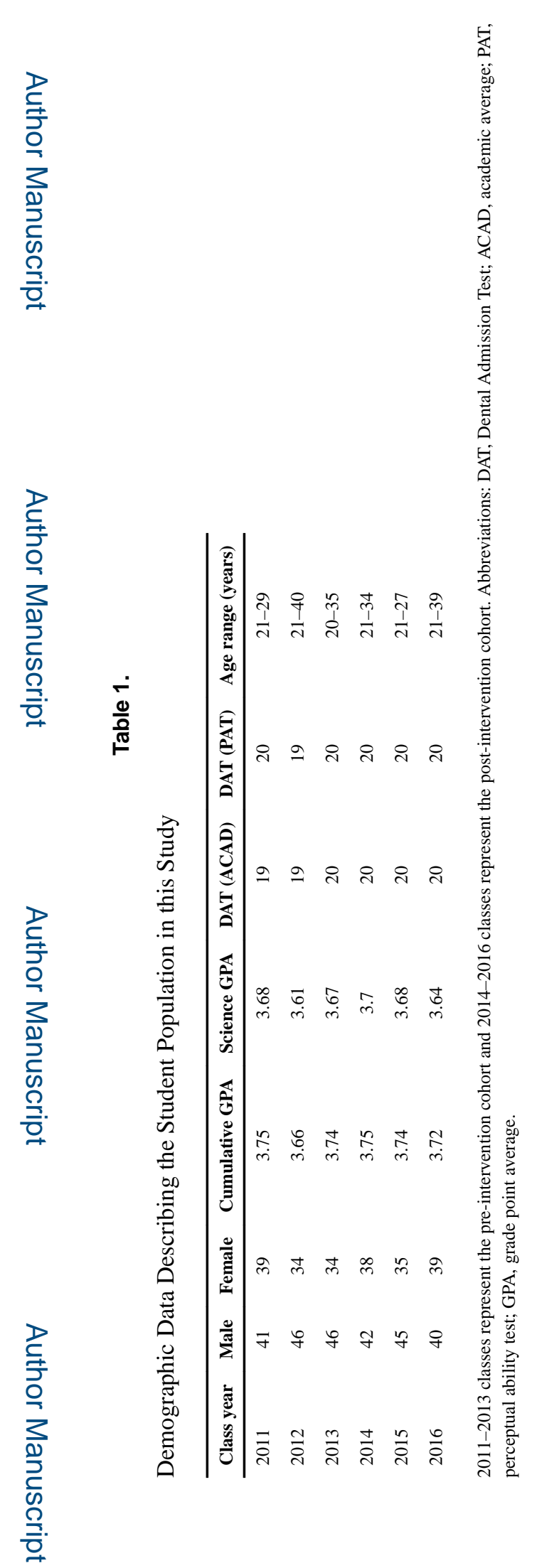

Anat Sci Educ. Author manuscript; available in PMC 2019 February 20. 


\section{을 \\ }

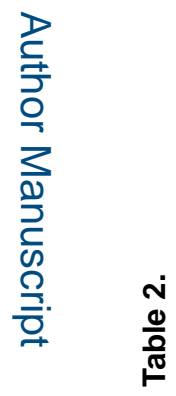

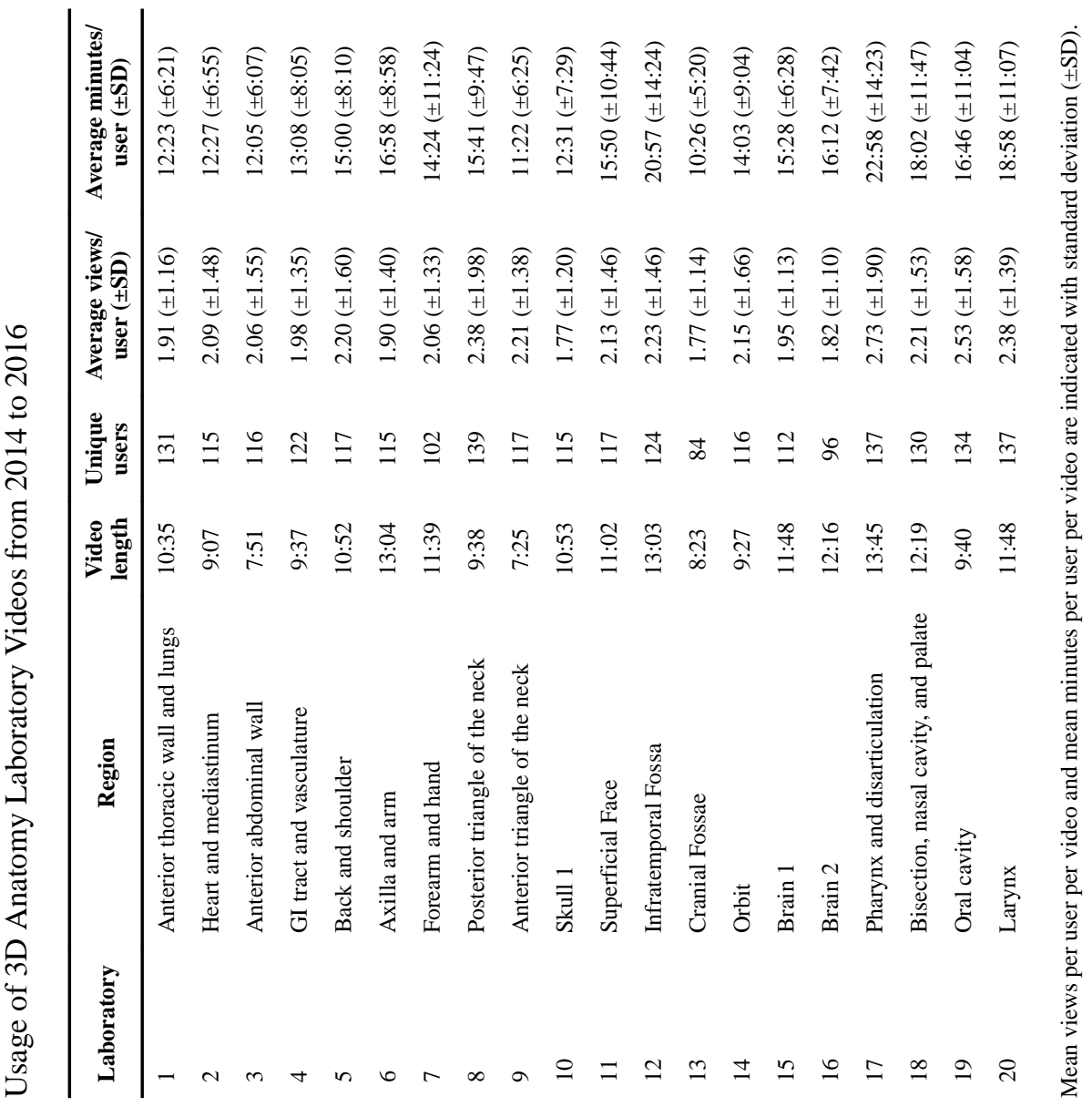

를

을 Anat Sci Educ. Author manuscript; available in PMC 2019 February 20. 


\section{롤 \\ 길}

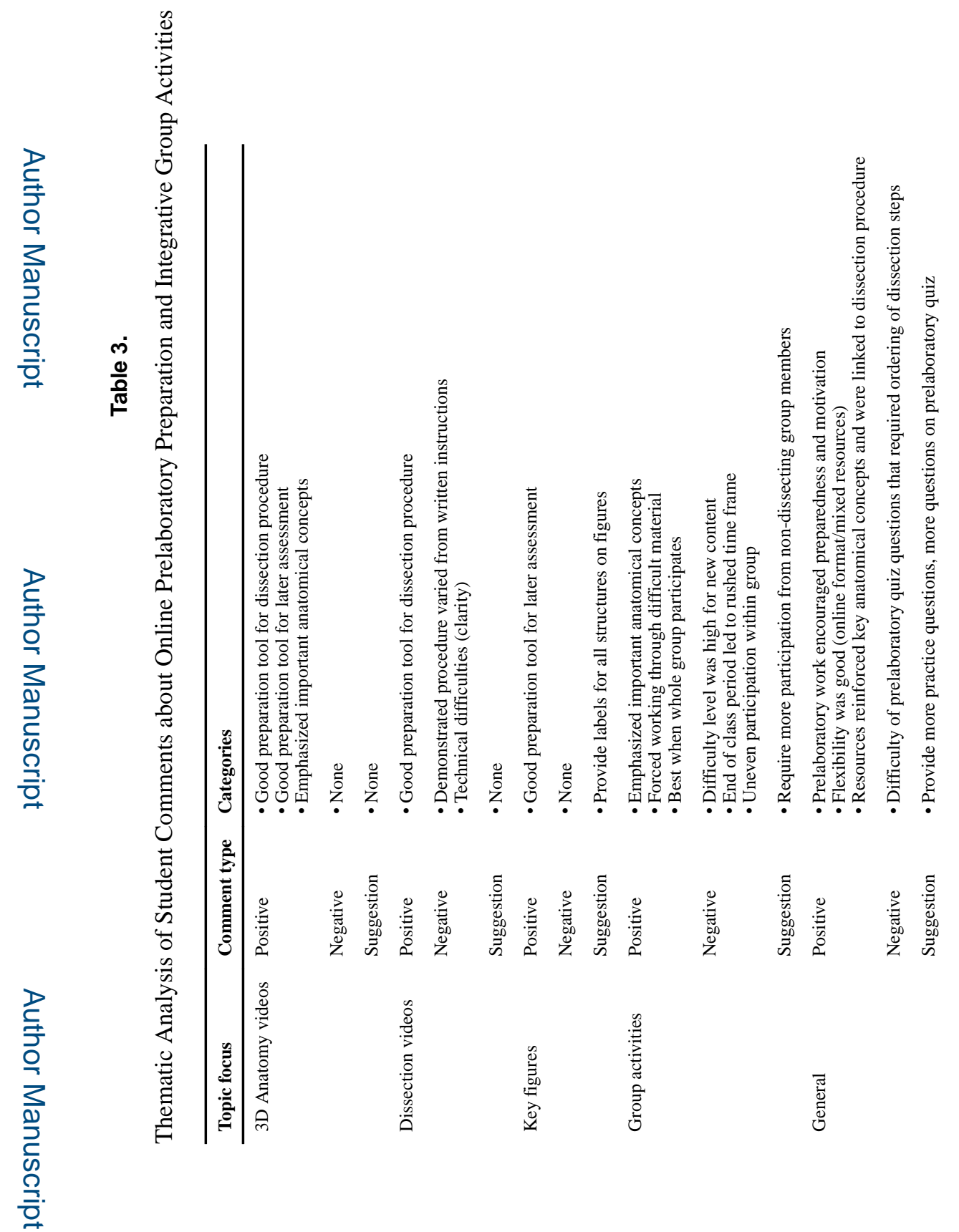


Table 4.

Performance on Laboratory Examinations Before and After Curriculum Change

\begin{tabular}{|c|c|}
\hline Cohort or sub-cohort & Examination mean $( \pm$ SD) \\
\hline \multicolumn{2}{|c|}{ Examination 1 (trunk, upper limb, neck) } \\
\hline Pre-flip (2011 sub-cohort) & $83.75( \pm 8.71)$ \\
\hline Pre-flip (2012 sub-cohort) & $89.36( \pm 6.91)$ \\
\hline Pre-flip (2013 sub-cohort) & $84.13( \pm 10.67)$ \\
\hline Total pre-flip (2011-2013 cohorts) & $83.38( \pm 10.77)^{a}$ \\
\hline Post-flip (2014 sub-cohort) & $86.19( \pm 10.10)$ \\
\hline Post-flip (2015 sub-cohort) & $86.36( \pm 10.80)$ \\
\hline Post-flip (2016 sub-cohort) & $79.06( \pm 13.53)$ \\
\hline Total post-flip (2014-2016 cohorts) & $83.88( \pm 12.02)^{a}$ \\
\hline \multicolumn{2}{|c|}{ Examination 2 (head and neuroanatomy) } \\
\hline Pre-flip (2011 sub-cohort) & $86.54( \pm 9.43)$ \\
\hline Pre-flip (2012 sub-cohort) & $87.38( \pm 8.72)$ \\
\hline Pre-flip (2013 sub-cohort) & $80.83( \pm 10.43)$ \\
\hline Total pre-flip (2011-2013 cohorts) & $88.93( \pm 8.04)^{b}$ \\
\hline Post-flip (2014 sub-cohort) & $86.59( \pm 9.30)$ \\
\hline Post-flip (2015 sub-cohort) & $89.91( \pm 9.17)$ \\
\hline Post-flip (2016 sub-cohort) & $87.59( \pm 9.30)$ \\
\hline Total post-flip (2014-2016 cohorts) & $88.04( \pm 9.32)^{b}$ \\
\hline \multicolumn{2}{|c|}{ Examination 3 (Head and Neck comprehensive) } \\
\hline Pre-flip (2011 sub-cohort) & $79.85( \pm 12.75)$ \\
\hline Pre-flip (2012 sub-cohort) & $90.06( \pm 8.20)$ \\
\hline Pre-flip (2013 sub-cohort) & $85.96( \pm 8.30)$ \\
\hline Total pre-flip (2011-2013 cohorts) & $83.64( \pm 10.03)^{c}$ \\
\hline Post-flip (2014 sub-cohort) & $88.03( \pm 9.26)$ \\
\hline Post-flip (2015 sub-cohort) & $85.28( \pm 9.01)$ \\
\hline Post-flip (2016 sub-cohort) & $83.22( \pm 11.80)$ \\
\hline Total post-flip (2014-2016 cohorts) & $85.51( \pm 10.25)^{c}$ \\
\hline \multicolumn{2}{|c|}{$\begin{array}{l}\text { Entire pre- and post-flip cohorts were compared by unpaired independent Student's } t \text {-test. Sub-cohorts from each year in the study were also } \\
\text { analyzed to examine variability from year to year; }\end{array}$} \\
\hline \multicolumn{2}{|c|}{${ }^{a} P$-value ( $t$-test) between these values was statistically non-significant $(P=0.629)$} \\
\hline \multicolumn{2}{|c|}{${ }^{b} P$-value $(t$-test) between these values was statistically non-significant $(P=0.264)$} \\
\hline
\end{tabular}

